\title{
Relibility Analysis of Indicator Functions in an Automated Multiscale Measuring System
}

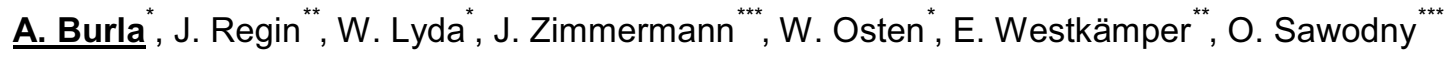 \\ *Institut für Technische Optik , Universität Stuttgart, Pfaffenwaldring 9, 70569, Stuttgart. \\ ** Institut für Industrielle Fertigung und Fabrikbetrieb, Universität Stuttgart, Nobelstr. 12, 70569 Stuttgart. \\ *** Institut für Systemdynamik, Universität Stuttgart, Pfaffenwaldring 9, 70569, Stuttgart.
}

\section{Introduction}

Modern micro systems are manufactured in batch processes at wafer scale with critical functional components in sub-micron dimensions. To ensure high quality of these components high resolution inspection systems are needed. Due to the limited area-related resolution of commercial available sensors a conflict between measurement time, object size and resolution arises.

In former publications we have presented the general design of an Automated Multiscale Measuring System (AMMS) based on an intelligent and hierarchical measurement strategy to balance this conflict [1, 2]. The system pursues a multiscale active exploration strategy, starting with a coarse scale sampling (fig. 1). To identify new regions of interest for more detailed measurements, task specific, coarse-scale indicator functions are used. Based on the information from the indicator analysis, sensory and positioning data are processed step-by-step and merged using intelligent data fusion. The system utilises high resolution and accuracy only where it is needed. By task specific sensor parameterisation and actuator control, the system automatically adapts to the given object and measurement task.

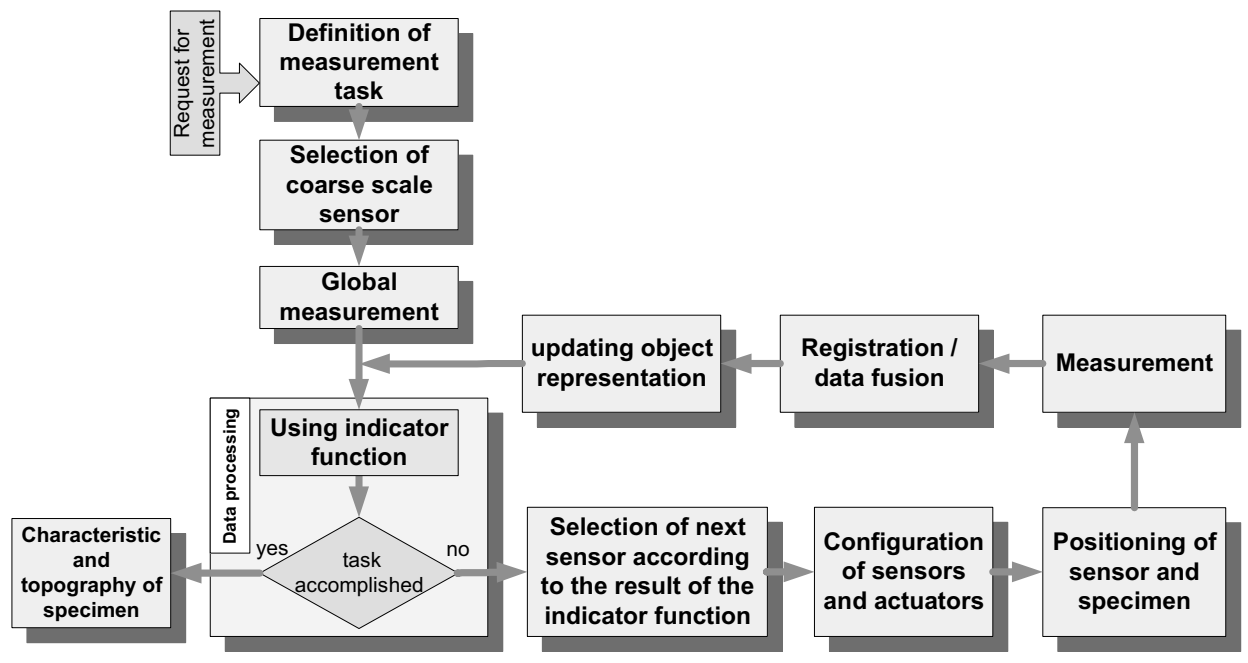

Fig. 1: Basic strategy of the AMMS

The concept was realised in a prototype based on a modified Mahr MFU 100. The prototype is armed with three different sensors for fast inspection of micro optical elements like micro lens arrays. Due to the fact that micro lens arrays are manufactured in batch processes, a wide bandwidth of defects from wide area pollution and missing lenses down to sub- $\lambda$ wavefront defects can occur. Hence the micro lens arrays are suitable objects for the investigation of measurement certainty and defect detection reliability of the AMMS.

This contribution is focused on the investigation of indicator reliability, a crucial performance factor for the multiscale measurement system. Several indicator algorithms are developed to accurately detect flaws and defects on the surface of the micro lens arrays. For the reliability analysis of the algorithms microlens arrays with different surface and shape defects are simulated using synthetic data based on mathematical as well as statistical methods.

The feature detection algorithms include Fourier self-filtering, two-point statistical texture featuring, scratch detection and normalized cross correlation. These algorithms are parameterised according to the type of defect. Reliability analysis of the detection algorithms was carried out to evaluate the amount of false positives or false negatives detected, to find the detection certainty of the algorithms with respect to the changes in the defect properties. The results of this reliability analysis are shown in the following sections. 


\section{Prototype implementation}

The concept, purpose and general design of an Automated Multiscale Measuring System (AMMS) was presented in [1]. Multiple optical sensors with different measurement ranges, resolutions and fields are combined to characterise extensive objects with micro and nano scale structures. The indicator functions serve as a connection between the scales thus allowing a consistent measurement process involving the desired sensors with the corresponding parameterisation. Sensor data fusion generates a consistent representation of the measured object in multiple scales. The main advantages of the AMMS lie in the fully automated process and in the reduced measurement time due to an adaptive strategy based on indicator functions and combined conditioning of sensors and model based control system. This time reduction and robustness make the AMMS suitable for production related measurement and inspection tasks.

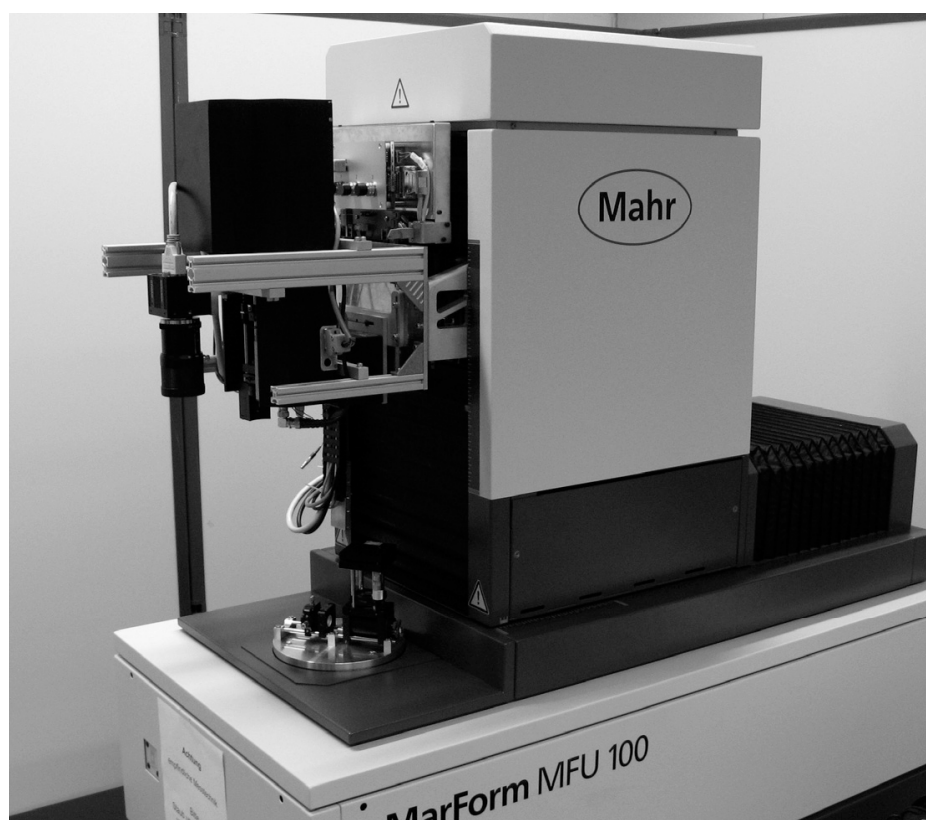

Fig. 2: AMMS prototype for fast microlens characterisation

The AMMS prototype is based on a modified Mahr MFU 100, directly controlled by a dSpace system, with an overall operating volume of $200 \mathrm{~mm} \times 300 \mathrm{~mm} \times 360^{\circ}$. To pursue a multiscale exploration the AMMS is equipped with three different sensors. The first sensor attached to the MFU is a video microscope, which has a measurement field of $18 \times 13 \mathrm{~mm}^{2}$ and a lateral resolution of $13 \mu \mathrm{m}$. A special lightning system with multiple light sources is installed, providing light for micro lens spot observation and scattered light analysis. In the next finer scale a confocal microscope for topographic measurements with lateral resolution between $2 \mu \mathrm{m}$ and $15 \mu \mathrm{m}$ is available. The finest scale is realised with a SiScan Sc 400 . It is utilized for high resolution measurements of restricted sub-regions.

The proposed model-based control system [5] is adjusted automatically with respect to the measurement strategy, sensors and positioning equipment, based on internal models and previous measurements. Therefore, the dynamic properties of sensors and data post-processing as indicator functions must be taken into account.

\section{Defect and indicator}

As stated in the introduction, the AMMS uses special indicators to select sub-regions for finer measurements. Indicators are deviations form the expected measurement results, giving a hint for an unresolved defect on the specimen in the actual sensor scale. For an exact classification of the possible defect, further measurements in finer scales are needed.

For the purpose of evaluating the multiscale strategy within the context of inspecting microlens arrays, three distinct defect types are considered: 1) point-like defects, such as minute particles or dust speckle, 2) one-dimensional defects, including cracks, scratches and fine fibres and 3) irregularities in the shape and size of the microlenses, including missing or partially missing microlenses. The defect types are 
chosen to be a representative set of surface defects. Sample measurements taken with the video microscopy from each category are shown in (Fig. 3).

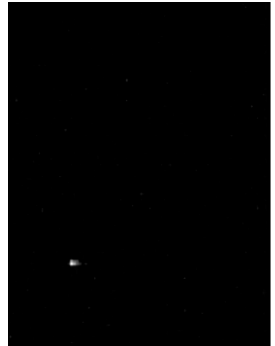

a)

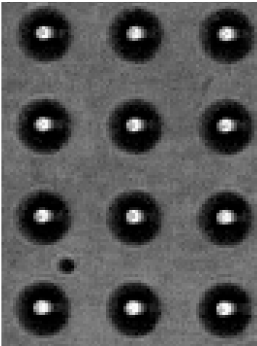

b)

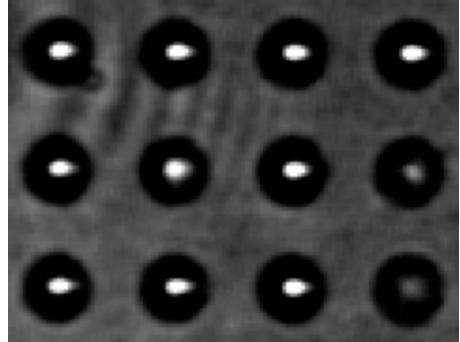

c)

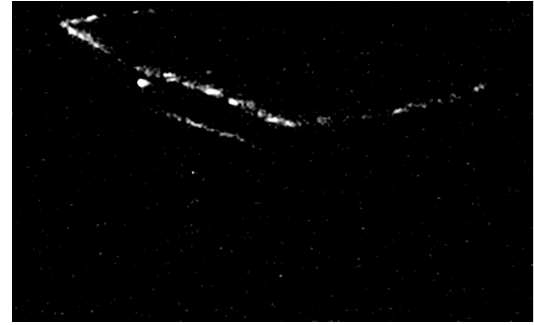

d)

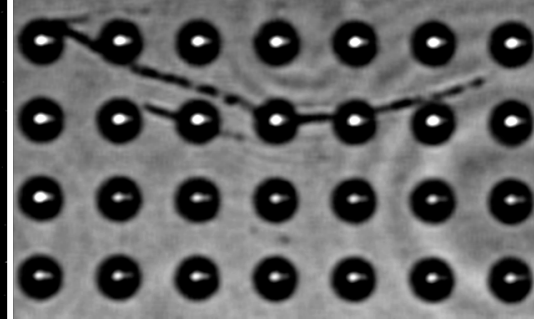

e)

Fig. 3: Measurement images taken by video microscopy of point defects in transmission (a) and reflection (b), defect microlenses in spot observation (c), line defects in transmission (d) and reflection (e)

Different defects lead to deviations from the predicted measurement results depending on the sensor type and specification. As mentioned before, these deviations are used for defect indication. This representation changes according to the defect types. For example from the video microscopy images in (Fig 3) it can be inferred that point like defects and line defects show variations in intensity with respect to the neighbouring pixels. Where as the shape defect's indicators depend on the type of shape defect in our case, for example from figure $3 c$ we can see that deformed lenses form faded or defocused spots.

\section{Defect Simulation}

Given the low number of observed defects in real production samples and the challenge of purposefully inducing defects ex post in a reproducible way, simulated defects offer several advantages over measurements of actual defects. Most important is a larger set of sample defects is achievable, and also fine-grained control of defect parameters and positions is possible.

The computer models used for defect simulation are motivated by observing the characteristics of real defects. For instance, from the video microscopy images in (Fig 3) it can be inferred that point-like defects appear as dark spots in transmission illumination and as bright spots in reflective illumination. Shape and structure are not discernible, since defect dimensions are close to the resolution limit of the video microscope. Hence, a simple defect model consisting of a Gaussian intensity peak is chosen, using height, width and position of the peak as the model parameters. The position of the defects was varied over the whole object field. The radius of the modelled point defects range from 1 to 10 pixels and the simulated intensity of the defect was varied from $10 \%$ to $80 \%$ of the background intensity in transmissive illumination and vice versa.

Similarly line defects appear darker in the transmission illumination and brighter in reflective illumination. Polynomial curves with variable thickness from 1 to 20 pixels, length chosen from 25 to 300 pixels and intensity variations similar to point defect simulation are used to model these defects, adding the polynomial coefficients to the model parameters (Fig. 4b).

Ideal micro lenses form perfect Gaussian spots on their focal plane when illuminated with a collimated light source. Irregularly shaped lenses form faded or defocused spots with reduced intensity. A video microscopy measurement of microlenses spots acquired with the AMMS is shown in Fig. 3c. In order to obtain simulated images of the spots generated by deformed or broken lenses, Zernike coefficients are computed based on a simulation in Zemax (Fig. 4d). The resulting wave front defects range from 0 to $3 \lambda$. 


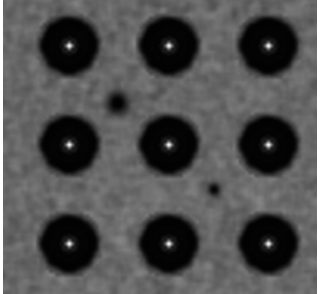

a)

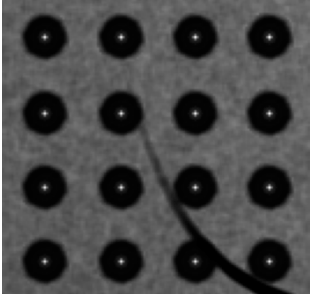

b)

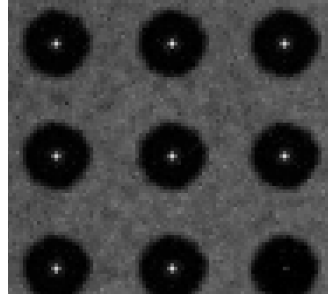

C)

Fig. 4: Synthetic measurement data of point defects (a), a scratch (b), microlenses in spot observation (c)

The defect models are applied to images of microlens arrays, thus generating synthetic measurement data, which serve as test cases for the indicator strategy.

\section{Defect and indicator detection algorithms}

To detect defect indicators in the synthetic measurement data, different indicator detection algorithms are used. Our major algorithms include Fourier self-filtering, two-point statistical texture featuring, scratch detection using region growing and normalized cross correlation.

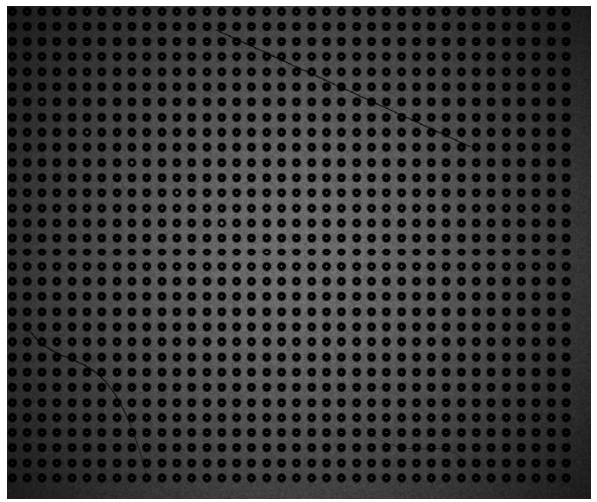

a)

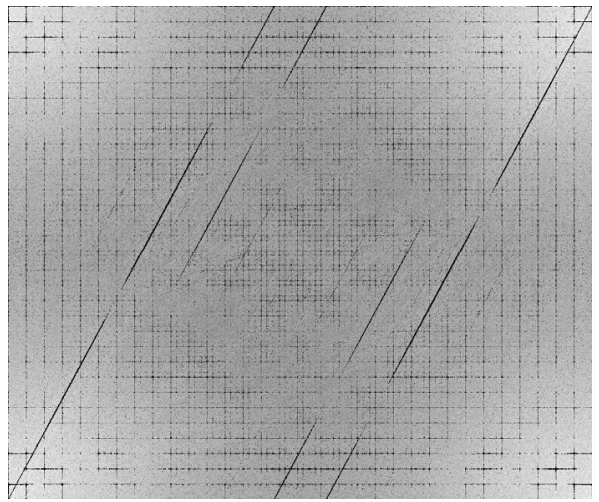

c)

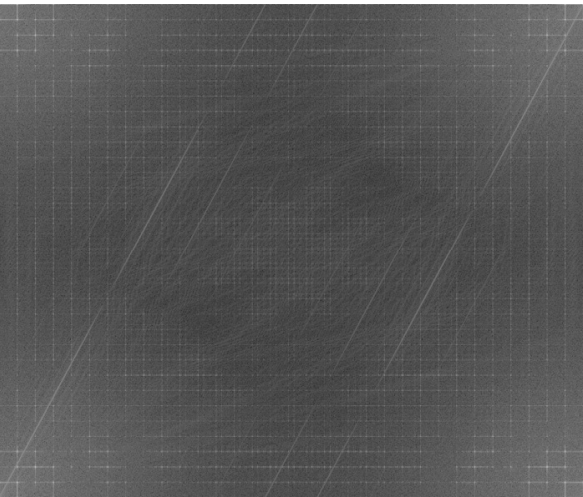

b)

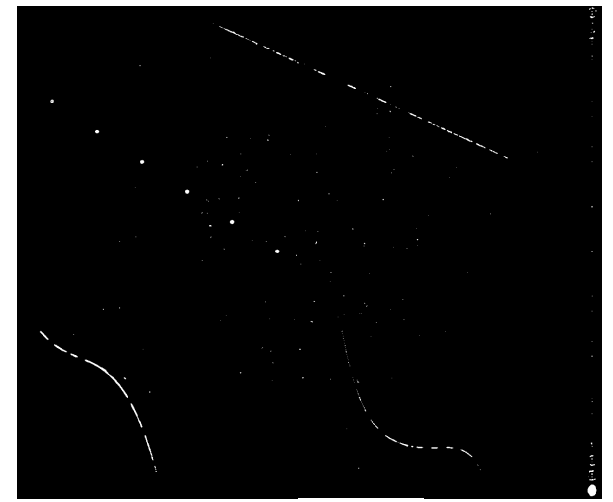

d)

Fig. 5: synthetic measurement data of spot observation by video microscope (a), FFT of the data (b), filtered FFT of the data (c), evaluation result of measurement data (d)

Fourier self-filtering makes use of the highly periodic nature of the microlens array images. By masking out the major peaks in the frequency domain, the periodic components of the image are removed.

Subsequently, the remaining non-periodic components are thresholded to get the absolute positions of the indicators. Two-point statistical texture featuring evaluates the surface texture of the microlens array. In general, technical as well as natural surfaces exhibit a characteristic surface structure (texture) permitting their detection and classification. Texture features and methods for surface inspection [6] are here used for the purpose of defect indication. The scratch detection algorithm uses edge detection with local thresholding for feature extraction and region growing for segmentation. 
Normalised cross-correlation compares the synthetic image with a single ideal microlens, acting as a template. The shape of the correlations peaks indicates the goodness of the lenses. The ideal lenses are segmented out from the original to obtain the defective lenses, point defects and line defects on the surface.

\section{Reliability analysis}

The reliability of the indicators is analysed using the synthetic images described above. Each test case contains a series of images, obtained by varying one of the defect parameters. The output of a feature detection algorithm is a binary classification of each pixel in the input image. In order to evaluate the correctness of the classification, the image is divided into square segments, 8 by 8 pixels in size. Each segment which contains one or more positive pixels is considered positive, the remaining segments negative. Ground truth is similarly given by the defect positions used in the simulation of the synthetic image.

To measure the performance of the indicator evaluation function, the sensitivity rate of the binary classification is computed. The sensitivity rate (sometimes referred to as recall rate) is the proportion of actual positives which are correctly identified, i.e. the number of true positives divided by the sum of true positives and false negatives. Similarly, the proportion of actual negatives which are correctly identified is denoted specificity. It is computed by dividing the number of true negatives with the sum of true negatives and false positives. High sensitivity is crucial for a good indicator function method, since all potential defects need to be indicated at the first scale level of the AMMS.

Exemplary evaluation of one test case is shown. In our setup, a video microscope with limited resolution of $13 \mu \mathrm{m}$ corresponding to one camera pixel is considered and the results from this sensor are simulated and used for reliability analysis of the functions. A line defect's indicator of varying thickness and shape is simulated. The results of that indicator detection are shown in figure 6.

As anticipated, the sensitivity increases with the scratch thickness. The curves for Fourier self-filtering, scratch detection and normalized cross correlation follow each other closely, with $80 \%$ sensitivity or more for scratches wider than 1 pixel. The texture base feature detection reaches the same level of sensitivity for scratches thicker than 6 pixels.

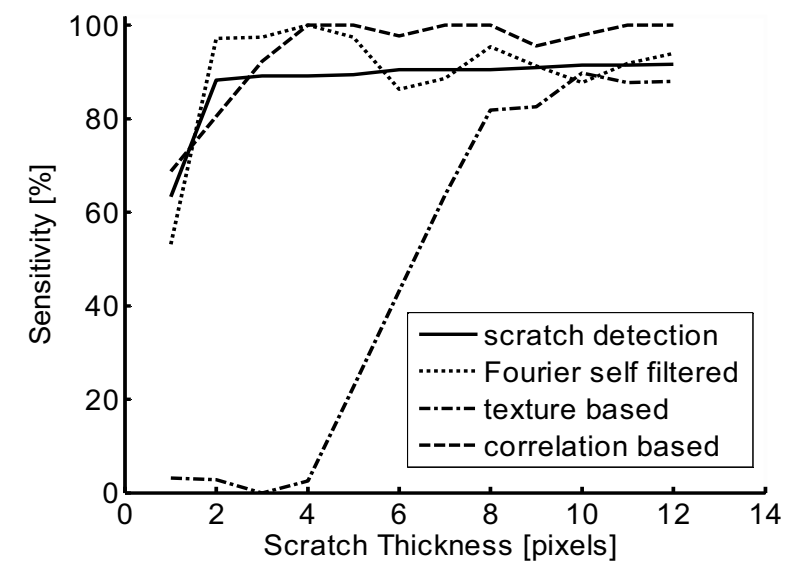

a)

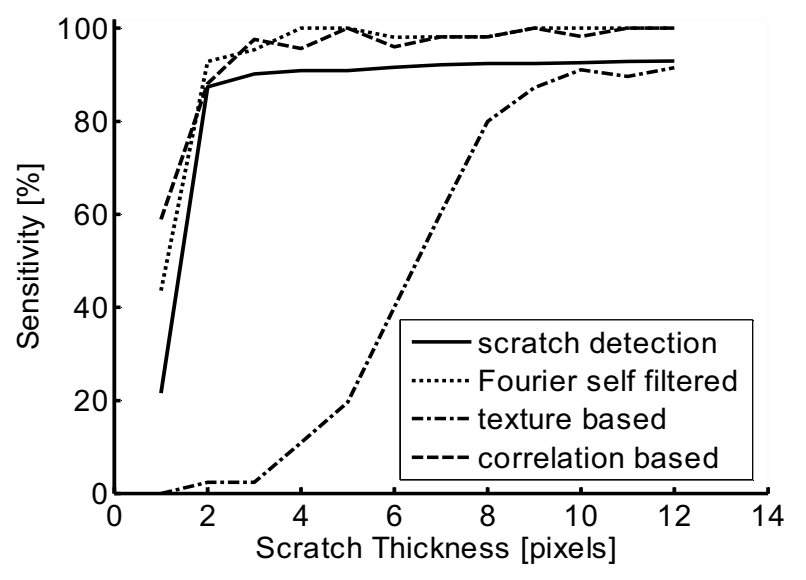

b)

Fig. 6: Sensitivity analysis of the indicator functions applied to linear (a) and curved scratches (b) of varying thickness and with an intensity of $25 \%$ of the background intensity.

The parameters of the indicator detection algorithms were set manually. Future work will address the optimisation of these parameters.

\section{Conclusion}

Reliability analysis was performed on different functions using microlens arrays to detect the three kinds of defects that are point defects, line defects and shape defects. Results of such experiments to detect 
line defects are shown in the previous section. It can be concluded from the graphs that the correlation based method, the scratch detection method and Fourier self filtering are better suited methods to detect line defects which are at least 1-2 pixels also depending on the defect contrast which should at least be $20 \%$. Texture based methods can only be used if the scratches have a certain thickness of approximately 6 pixels or more. The investigation on the point defects shows that the defect contrast has to be more than $20 \%$ for a detection sensitivity of $80 \%$. Experimental measurements with our prototype AMMS show that a scratch with a thickness smaller than $5 \mu \mathrm{m}$ and a depth of $200 \mathrm{~nm}$ could be detected. This shows that defects with dimensions under the camera resolution of $13 \mu \mathrm{m}$ can be indicated.

Similar investigations have been performed for shape deviations, described by Zernike polynomials. It turns out that under video microscopy correlation based algorithms and Fourier self-filtering are sensitive to deviation greater than $\lambda / 2$. Better results should be possible with better camera dynamics and optimized algorithms parameters

Hence, Fourier self filtering is better suited for large scale sensors, thus for detecting the defects from video microscopy images, because of the high periodicity of the lenses. Correlation based methods are better suited for both video microscopy and even at finer scales like confocal microscopy images, due to the fact that it does not require high periodicity in the images. Hence, reliability analysis can be performed depending on the task specification, to determine suitable methods for defect detection at every scale.

\section{Summary}

An automatic multi-scale measurement system has been presented along with a method for evaluating the reliability of one of its key components: the indicator detection functions. Four distinct indicator algorithms for the first scale level were considered. A set of synthetic measurement data was generated based on models for point, line and lens shape defects. By varying the parameters of the defect models, sample sets for the indicator evaluation were generated. The reliability of the indicator functions was analysed by comparing known defect positions with detection function's output, and by computing the system's sensitivity.

With respect to our measurement system and the chosen periodically structured specimen (microlens arrays) the analysis results show that Fourier self filtering is an effective method at large scales and correlation based methods are also effective at finer scales. Scratch based methods are affective for surface scratch and point defects under reflective illumination. Texture based methods indicate defects irrespective of illumination and defect type at the cost of lower sensitivity.

Experimental results have also shown that the functions can be optimized or tuned to better suit the task specification.

This work was supported by the DFG under SPP-1159.

\section{References:}

[1] T. Wiesendanger, W. Osten, J. Pannekamp, J. Regin, E. Westkämper: Neue multiskalige Mess- und Prüfstrategien für die Produktion von Mikrosystemen In: Mikrosystemtechnik Kongress 2005. GMM, VDE, VDI, (eds.), VDE Verlag Berlin, Germany; pp 677-680

[2] J. Zimmermann, J. Regin, W. Lyda, T. Wiesendanger, O. Sawodny, E. Westkämper, W. Osten: Definition and Design of an Automated Multiscale Measuring System; euspen 2008, Zurich, Switzerland

[3] J. Regin, J. Neher, J. Pannekamp, E. Westkämper, T. Wiesendanger, W. Osten: Multiskalige Messstrategien für die Mikrosystemtechnik In: Informationsfusion in der Mess- und Sensortechnik, VDI/VDE-GMA, Karlsruhe, Germany: Universitätsverlag 2006, pp 137-143

[4] E. Westkämper, W. Osten, J. Regin, T. Wiesendanger: Multiskalige Mess- und Prüfstrategien in der Mikro- und Nanomesstechnik In: Messtechnik für Mikro- und Nano-Engineering Düsseldorf, VDI/VDE-GMA, Germany: VDI Verlag, 2006, pp 141-150

[5] J. Zimmermann, O. Sawodny: Modeling for Simulation and Control of a X-Y High Precision Positioning Table. In: Proceesings of IEEE CASE 2007, September 22-25, 2007, Scottsdale, Arizona, USA

[6] J. Pannekamp: Adaptive Verfahren zur Bewertung texturierter Oberflächen, Heimsheim : Jost-Jetter Verlag, 2005.

(IPA-IAO Forschung und Praxis; 414). (Stuttgart, Univ., Fak. Maschinenbau, Inst. für Industrielle Fertigung und Fabrikbetrieb, Diss. 2004). - ISBN 3-936947-53-8. - URN urn:nbn:de:bsz:93-opus-22883 